\title{
Ion mass effect in plasma-induced charging
}

\author{
Gyeong S. Hwang and Konstantinos P. Giapis \\ Division of Chemistry and Chemical Engineering, California Institute of Technology, Pasadena, \\ California 91125
}

(Received 20 June 1997; accepted for publication 30 July 1997)

\begin{abstract}
Simulations of charging and profile evolution during etching of high aspect ratio polysilicon gates in typical high-density plasmas containing heavy ions (e.g., $\mathrm{BCl}_{3}^{+}, \mathrm{Cl}_{2}^{+}$), predict a reduction in charging and notching when lighter ions (e.g., $\mathrm{He}^{+}$) are added. The reduction occurs because of the influence of the ion mass on the ratio $R$ of the ion sheath transit time to the rf period, which determines the spread in the ion energy distribution at the wafer. The effect is most pronounced when $R \leqslant 0.1-0.2$ for light ions and, simultaneously, $R \geqslant 0.6$ for heavy ions; then, more light ions arrive at the patterned structure with low energies, where they help decrease localized charging. When the rf bias frequency is reduced so that $R \leqslant 0.3$ for all ions, the effect disappears. (C) 1997 American Institute of Physics. [S0003-6951(97)04639-1]
\end{abstract}

Mixtures of gases are frequently used in plasma etching; ${ }^{1}$ for example, a $\mathrm{Cl}_{2} / \mathrm{BCl}_{3}$ plasma is widely used in metal etch, while $\mathrm{HBr} / \mathrm{Cl}_{2}$ is employed in polysilicon gate etching. The mixture gets more complicated during the overetch step, when inert (e.g., He) and/or etch inhibitor gases (e.g., $\mathrm{O}_{2}$ ) are added to improve plasma stability and facilitate profile control. ${ }^{1}$ The role of chemistry on plasma etching is of paramount importance, albeit extraordinarily complex given the number of ions and neutrals involved and the multitude of phenomena occurring during the plasmasurface interaction. ${ }^{2}$ Starting with a $\mathrm{He} / \mathrm{Cl}_{2} / \mathrm{BCl}_{3}$ plasma, for example, one can expect a number of ions to be present: $\mathrm{He}^{+}, \mathrm{Cl}^{+}, \mathrm{Cl}_{2}^{+}, \mathrm{BCl}_{3}^{+}, \mathrm{BCl}_{2}^{+}, \mathrm{BCl}^{+}$, and $\mathrm{B}^{+}$, in addition to any possible negative ions. ${ }^{3}$ The etching behavior of the various ions is usually discussed in terms of differences in reactivity. For ion-limited etching, the etch yield depends strongly on ion energy. ${ }^{4}$ Thus, the corresponding ion energy distribution function (IEDF) at the wafer determines the contribution of a particular ion to etching.

All positive ions are accelerated in the sheath, subject to the same electric fields. According to sheath theory, ${ }^{5}$ ions enter the sheath with the Bohm velocity, $\mathrm{v}_{B}$ $=\left(k_{B} T_{e} / M_{i}\right)^{1 / 2}$, where $k_{B}$ is the Boltzmann constant, $M_{i}$ is the ion mass, and $T_{e}$ is the electron temperature. Thus, the presence of widely disparate ion masses in the plasma will cause differences in the ion sheath transit time, $\tau_{i o n}$. The ratio $R$ of $\tau_{i o n}$ to the rf period determines the spread in the IEDF. ${ }^{6}$ When $R \leqslant 0.2$ or $R>2.0$ for all ions, the differences in $\mathrm{v}_{B}$ will not influence the IEDF of the various ions. However, when the rf period is such that $R=0.1-0.2$ for lighter ions but $R \geqslant 0.6$ for heavier ions, the energy spread in the IEDFs will be markedly different. ${ }^{6}$ This special case actually occurs often in typical high-density $\left(10^{11}-10^{12} \mathrm{~cm}^{-3}\right)$ plasmas biased at $13.56 \mathrm{MHz}$, where lighter ions (e.g., $\mathrm{H}^{+}, \mathrm{He}^{+}$, $\mathrm{B}^{+}$) cross the sheath in a small fraction of the rf cycle, in contrast to much heavier ions (e.g., $\mathrm{Cl}_{2}^{+}, \mathrm{BCl}_{3}^{+}$) that require almost a full cycle. In general, the IEDF of heavier ions will have a smaller spread of translational energies. Lighter ions crossing during the sheath potential minimum will attain much reduced energies. While these differences are relatively unimportant when etching flat wafers, they can influence plasma-induced charging in patterned areas.

A convenient way to study charging effects is through "notching," a wedgelike sidewall profile irregularity which appears at metal or poly-Si gates right above the underlying $\mathrm{SiO}_{2}$ during overetching. ${ }^{7}$ Tabara et al. ${ }^{8}$ compared various metal etch recipes in terms of notching and reported that the presence of heavy ions, such as $\mathrm{BCl}_{3}^{+}$or $\mathrm{BCl}_{2}^{+}$, in the plasma caused more severe notching due to their ability to remove the sidewall protecting film more easily than lighter ions. Addition of $\mathrm{He}$ to a $\mathrm{Cl}_{2} / \mathrm{BCl}_{3}$ plasma did decrease notching; however, it remained unclear whether this was due to the reduced abundance of heavy ions or the reduced photoresist selectivity and the concomitant decrease in aspect ratio. ${ }^{9}$ When using an $\mathrm{HCl}$ plasma, they found a $30 \%$ reduction in notching over a $\mathrm{Cl}_{2} / \mathrm{BCl}_{3}$ plasma. Remarkably, the addition of $\mathrm{He}$ to the $\mathrm{HCl}$ plasma reduced notching dramatically without loss of selectivity; no reasonable explanation for this effect was offered. ${ }^{10}$ The diluting effect of He should be discounted because the overetch time-during which the notch forms - was a fixed percentage of the main etch, which was also prolonged. ${ }^{8}$ A slight increase in the measured electron temperature $(10 \%-20 \%)$ should actually have worsened notching. ${ }^{11} \mathrm{We}$ shall show that addition of the (light mass) He helps decrease local charging through its influence on the IEDF, which consistently explains the results of Tabara et al.

The complexity of the $\mathrm{He} / \mathrm{Cl}_{2} / \mathrm{BCl}_{3}$ plasma and the lack of data on the energy and angular dependence of the reactivity of the various ions at the metal or poly-Si surface make the simulation of charging and etching an impossible task at the present time. ${ }^{2}$ Nevertheless, since we wish to only make a point about ion mass effects, it is instructive to compare two simple situations: ${ }^{12}$ plasma $\mathrm{X}$, consisting of $\mathrm{He}^{+} / \mathrm{Cl}^{+} / \mathrm{BCl}_{3}^{+}=0.0: 0.5: 0.5$, and plasma $\mathrm{Y}$, consisting of $\mathrm{He}^{+} / \mathrm{Cl}^{+} / \mathrm{BCl}_{3}^{+}=0.5: 0.25: 0.25$. Other plasma parameters are taken to be: electron density of $5 \times 10^{11} \mathrm{~cm}^{-3}, T_{e}=4.0 \mathrm{~V}$, $T_{i}=0.5 \mathrm{~V}$. The wafer electrode is biased; the rf bias imposed across the sheath is given by $V_{r f} \sin \omega t$, where $\mathrm{V}_{r f}=30 \mathrm{~V}$ and $\omega=13.56 \mathrm{MHz}$. We will compare charging and notch evolution during overetching of poly-Si gate electrodes ${ }^{13}$ in the two hypothetical plasmas. The pattern consists of many isolated $0.3 \mu \mathrm{m}$ lines separated by $0.3 \mu \mathrm{m}$ spaces (trenches). Identical patterns are separated by open areas with a width of $4 \mu \mathrm{m}$. At the onset of overetching, each feature consists of a $0.9 \mu \mathrm{m}$ photoresist mask (insulating) onto $0.3 \mu \mathrm{m}$ $\mathrm{n}^{+}$-poly-Si gates, formed on top of a layer of $\mathrm{SiO}_{2}$, thick 


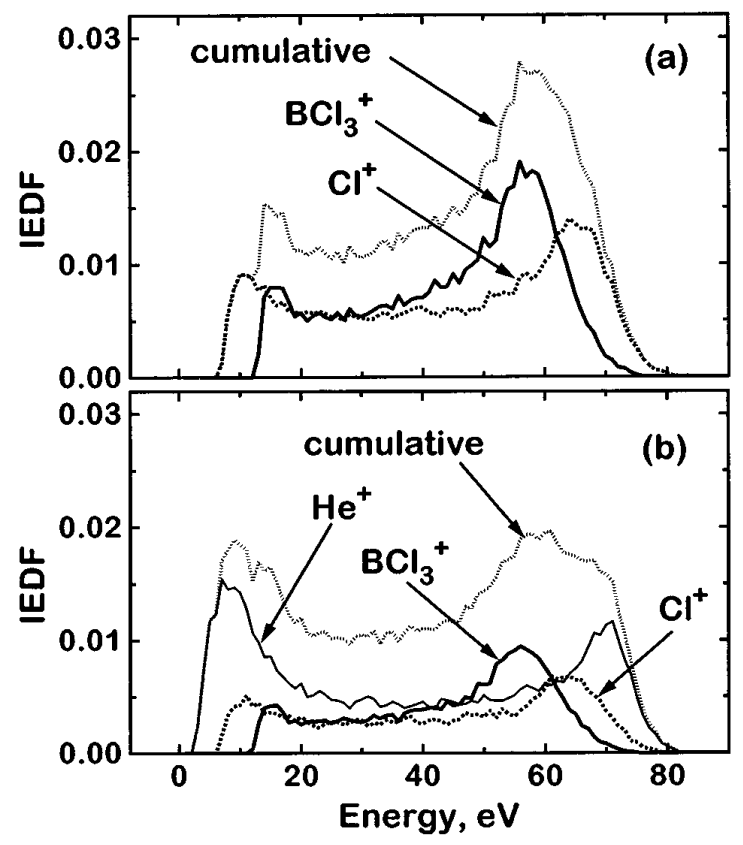

FIG. 1. Ion energy distribution functions for the various ions contained in two hypothetical plasmas: (a) plasma $\mathrm{X}\left(\mathrm{He}^{+} / \mathrm{Cl}^{+} / \mathrm{BCl}_{3}^{+}=0.0: 0.5: 0.5\right)$, and (b) plasma $\mathrm{Y}\left(\mathrm{He}^{+} / \mathrm{Cl}^{+} / \mathrm{BCl}_{3}^{+}=0.5: 0.25: 0.25\right)$. The cumulative energy distribution is also shown.

enough $(>100 \mathrm{~nm})$ to prevent electron tunneling.

The IEDFs at the wafer are calculated by Monte Carlo simulation of ion transport through the oscillating sheath. ${ }^{13}$ Ions enter the sheath with a corresponding Bohm velocity and are accelerated subject to a time-varying potential across the sheath, given by: ${ }^{5}$

$V_{s h}=V_{r f}(1+\sin \omega t)+\frac{T_{e}}{2}\left[\ln \left(\frac{M_{i}}{2 \pi M_{e}}\right)-\ln \left(\frac{2 \pi V_{r f}}{T_{e}}\right)\right]$,

where the second term represents the dc potential that spontaneously develops across the sheath $\left(M_{e}=\right.$ electron mass). When multiple ions are present, $M_{i}$ is calculated by summing the abundance-weighted ion masses. Thus, $\mathrm{V}_{s h}$ gets slightly shifted when the plasma composition changes. Since the ions enter the sheath at various phases of the rf cycle, they acquire a different energy as they cross the sheath, which makes the IEDFs bimodal. ${ }^{6}$ Figure 1(a) illustrates this effect for both $\mathrm{Cl}^{+}$and $\mathrm{BCl}_{3}^{+}$of plasma X. Note the larger spread in the IEDF of $\mathrm{Cl}^{+}$(the lighter ion). When large amounts of $\mathrm{He}$ are added to form plasma $\mathrm{Y}$, the contribution of $\mathrm{He}^{+}$to higher and, especially, lower energies is dramatic [Fig. 1(b)]. For the plasma conditions considered, the average $\mathrm{He}^{+}$transit time is $\approx 0.17$ of the rf period, whereas that for $\mathrm{BCl}_{3}^{+}$is $\approx 0.64$ of the same; this difference leads to a larger spread in the IEDF of $\mathrm{He}^{+}$. Indeed, the low and high energy peaks of its IEDF appear at $\sim 7$ and $71 \mathrm{eV}$, while those for $\mathrm{BCl}_{3}^{+}$appear at 17 and $57 \mathrm{eV}$, respectively. Because of its intermediate mass, the energy peaks for $\mathrm{Cl}^{+}$are between the respective values for $\mathrm{He}^{+}$and $\mathrm{BCl}_{3}^{+}$. Thus, the cumulative IEDF when $\mathrm{He}^{+}$is present has significantly broadened energy peaks.

Iterative Monte Carlo simulations of microstructure charging are performed ${ }^{13}$ as follows. Electrons and ions are generated successively above the patterned area with energy and direction determined by randomly sampling the corre- sponding energy and angular distributions. For multiple ions, sampling is weighted by their abundance in the plasma. Then, charged particles are followed as they impinge on, and transfer their charge to various surfaces. Charge deposition creates local electric fields determined by solving the Laplace equation. The fields are used to alter ion trajectories and are modified self-consistently as more charge accumulates. Steady-state charging is reached when the potential distribution along the bottom $\mathrm{SiO}_{2}$ surface no longer changes. ${ }^{13} \mathrm{~A}$ dynamic equilibrium is achieved, where electron and ion currents to various surface segments balance exactly. Surface currents and secondary electron emission are both neglected for reasons explained elsewhere. ${ }^{13}$

The steady-state charging potential distribution in the "edge" trench reveals the perturbation in the local ion dynamics occurring as a result of surface charging (Fig. 2). Gradients on this potential surface are a measure of the electric field that influences ion motion. As previously shown, ${ }^{13}$ an asymmetric potential distribution, with a pronounced peak near the inner sidewall foot of the edge line, is critical for notch formation. Such a potential surface is obtained for both plasmas $\mathrm{X}$ and $\mathrm{Y}$. While the potential peak at the bottom surface appears at the same location and has the same magnitude for the two plasmas, the potential surfaces away from the peak differ quantitatively. In the presence of He [Fig. 2(b)], the potential surface is "depressed" to lower values (by about 6-8 V), as compared to that developing for the plasma without He [Fig. 2(a)]. Note also that the potentials of the gates that confine the trench are likewise lower. The potential surface actually has a lower slope towards the edge line, which implies that the flux and energy of the deflected ions will both be slightly smaller. These observations suggest a reduction in notching when $\mathrm{He}$ is added to the plasma, even if $\mathrm{He}^{+}$had the same etch yield as $\mathrm{Cl}^{+}$.

When multiple ions bombard the chlorinated poly-Si surface, the reactivity with the surface depends on the chemical nature and energy of the ion. Furthermore, the ability of each ion to remove adsorbates from the surface depends on the collision cross-section which, in turn, depends on the ion mass and energy. Under ion-flux-limited conditions and low ion energies $(<100 \mathrm{eV})$, etching proceeds frequently by collision-induced desorption; etching commences when the ion energy exceeds a threshold value, $E_{t h}$, beyond which the etch yield depends on the square root of the energy times an ion-dependent prefactor. While values for these parameters have been proposed in the case of $\mathrm{Cl}^{+}$etching of poly-Si, ${ }^{13}$ they are generally unavailable for other ions, limiting the scope of profile evolution simulations. ${ }^{2}$ Nevertheless, it is still instructive to proceed by making the unrealistic assumption that the parameters for $\mathrm{He}^{+}$and $\mathrm{BCl}_{3}^{+}$are the same as for $\mathrm{Cl}^{+}$. Based on this assumption, notch profiles for each of plasmas $\mathrm{X}$ and $\mathrm{Y}$ have been generated by methods described in detail elsewhere, ${ }^{13}$ and are compared in Fig. 3 for two rf frequencies. At $13.56 \mathrm{MHz}$, the presence of $\mathrm{He}^{+}$in the plasma facilitates a reduction in notching, visible despite the unrealistic assumption that the etch yield for $\mathrm{He}^{+}$is the same as for $\mathrm{Cl}^{+}$. No effect on notching is seen at $0.4 \mathrm{MHz} .{ }^{14}$ Note that the expected lower reactivity of $\mathrm{He}^{+}$should result in a more significant reduction of notching in plasma $\mathrm{Y}$ than that shown in Fig. 3. These observations explain well the call for 

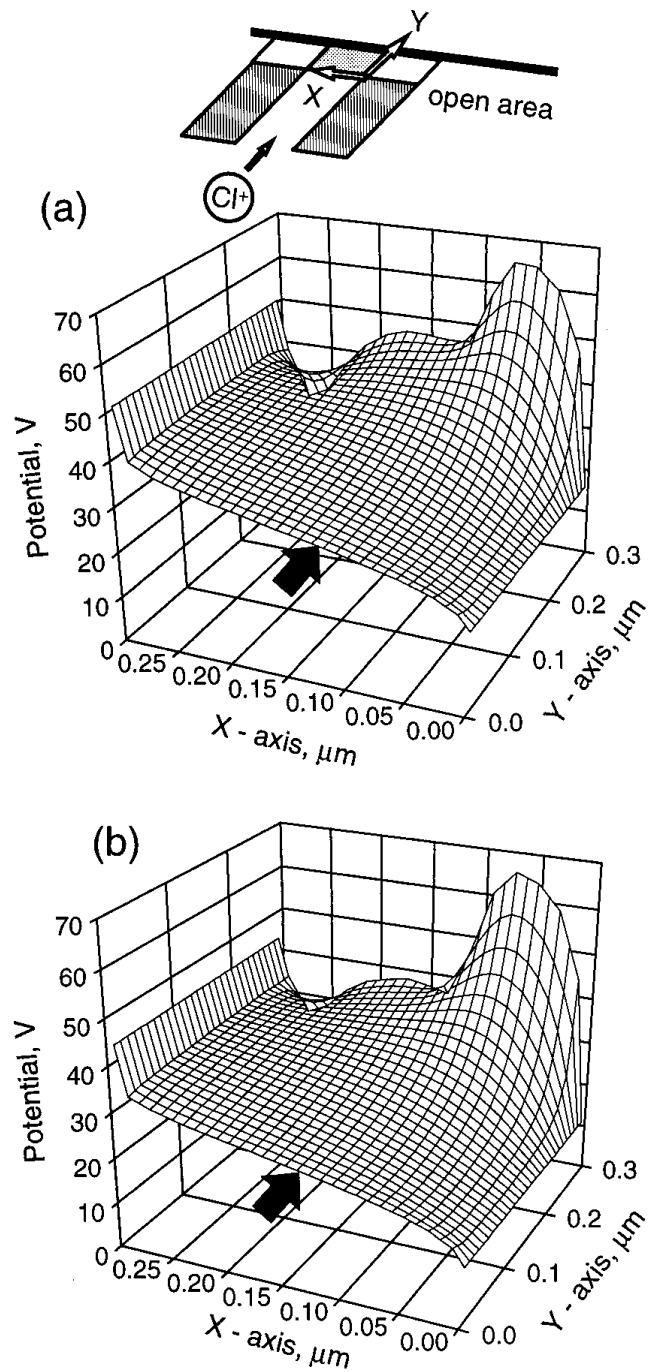

FIG. 2. Three-dimensional charging potential distributions in the "edge" trench of a dense pattern bordering an open area for (a) plasma $X$, and (b) plasma Y, after steady-state charging has been reached. The inset in (a) illustrates the area of interest and defines the origin for the potential surface. The microstructure has been rotated so that the direction of ions as they approach the potential surface corresponds to the direction of ions as they enter the trench (arrows).

He in metal and poly-Si overetch recipes. ${ }^{8}$

In conclusion, there exists an ion mass effect that influences charging during etching in high-density plasmas. The effect occurs in the presence of ions with widely disparate masses and only when the platen electrode is biased at frequencies that allow the lighter ions to cross the sheath in a small fraction of an rf cycle while the heavier ions require a lot more time. As a result, the IEDF of the lighter ions has a pronounced low energy component that is deflected at the trench bottom to neutralize the negatively charged upper photoresist sidewalls. More electrons can enter the trench, thus facilitating reduced in-trench potentials-and a concurrent decrease in the energy of ions that bombard the sidewalls. Thus, the light ion-mass effect emerges as the main reason for the reduction in notching that occurs with the addition of He to the plasma, rather than any heavy-ion dilution effects or scavenging of reactive radicals. ${ }^{8}$ The beneficial reduction in charging should become more evident with more light ions (e.g., $\mathrm{H}^{+}, \mathrm{He}^{+}, \mathrm{B}^{+}$) in the plasma, provided

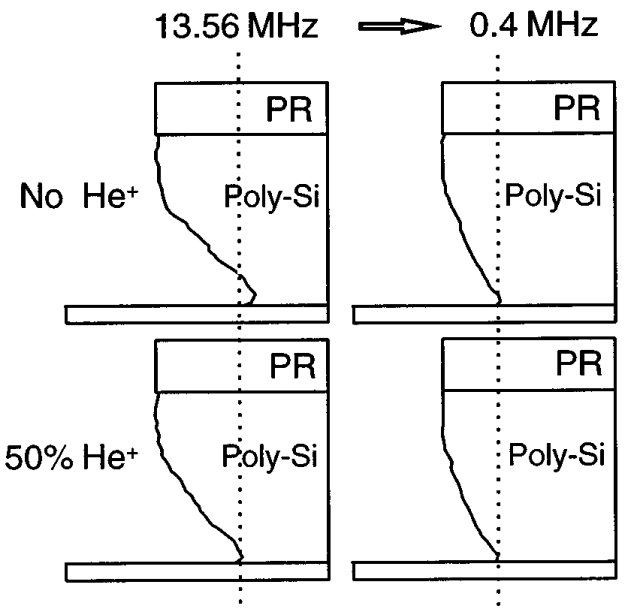

FIG. 3. Simulated notch profiles at the inner side of the outermost line, predicted for fixed (arbitrary) overetching in plasma $\mathrm{X}$ (no He) and plasma $\mathrm{Y}$ (with $\mathrm{He}$ ); profiles for $\omega=0.4 \mathrm{MHz}$ are also shown. The same etch yield dependence on incident energy and angle for all ions present was assumed (unrealistic); the notch is expected to be reduced-in the presence of $\mathrm{He}-$ more than the figure implies. The poly-Si aspect ratio has been preserved; however, the photoresist (PR) vertical dimension has been truncated to save space (true aspect ratio $=4: 1$ ).

the appropriate conditions for sheath thickness and rf bias frequency exist. ${ }^{14}$

This work was supported by a National Science Foundation-Career Award and a Camille Dreyfus TeacherScholar Award to KPG. An Applied Materials Scholarship in partial support of GSH is gratefully acknowledged.

${ }^{1}$ D. L. Flamm, in Plasma Etching: An Introduction, edited by D. M. Manos and D. L. Flamm (Academic, New York, 1989).

${ }^{2}$ D. B. Graves, M. J. Kushner, J. W. Gallagher, A. Garscadden, G. S. Oehrlein, and A. V. Phelps, Database Needs for Modeling and Simulation of Plasma Processing (National Academy Press, Washington, DC, 1996). ${ }^{3}$ Gas flow rates and electron impact ionization cross-sections influence the relative abundance of ions in the plasma for a given electron temperature, pressure, and plasma density.

${ }^{4}$ C. Steinbrüchel, Appl. Phys. Lett. 55, 1960 (1989).

${ }^{5}$ M. A. Lieberman and A. J. Lichtenberg, Principles of Plasma Discharges and Materials Processing (Wiley, Inc., New York, 1994).

${ }^{6}$ M. S. Barnes, J. C. Foster, and J. H. Keller, IEEE Trans. Plasma Sci. 19, 240 (1991).

${ }^{7}$ T. Nozawa, T. Kinoshita, T. Nishizuka, A. Narai, T. Inoue, and A. Nakaue, Jpn. J. Appl. Phys., Part 1 34, 2107 (1995).

${ }^{8}$ S. Tabara, Y. Kitayama, T. Hatakeyama, K. Katsuragi, and M. Tanage, Proceedings of the 2nd International Symposium on Plasma ProcessInduced Damage, Monterey, CA, 1997, p. 233.

${ }^{9}$ G. S. Hwang and K. P. Giapis, J. Appl. Phys. 82, 566 (1997).

${ }^{10}$ The reduction in notching for the $\mathrm{HCl} / \mathrm{He}$ plasma was attributed (Ref. 8) to "scavenging of excess $\mathrm{Cl}$ radicals by $\mathrm{H}$ radicals." It was not clarified why Cl-radical scavenging did not occur in the pure $\mathrm{HCl}$ plasma.

${ }^{11}$ G. S. Hwang and K. P. Giapis, J. Appl. Phys. 81, 3433 (1997).

${ }^{12}$ In reality, several other ions will be present depending on feedstock, pressure, and plasma power; see H. Kazumi and K. Tago, Proceedings of 3rd International Conference on Reactive Plasmas, Nara, Japan, 1997, p. 11. However, since $\mathrm{R} \propto M_{i}{ }^{1 / 2}$, the IEDF of $\mathrm{BCl}_{2}^{+}$and $\mathrm{Cl}_{2}^{+}$is virtually identical to that of $\mathrm{BCl}_{3}^{+}$; likewise, the IEDF of $\mathrm{B}^{+}$will not differ significantly from that for $\mathrm{He}^{+}$

${ }^{13}$ G. S. Hwang and K. P. Giapis, J. Vac. Sci. Technol. B 15, 70 (1997).

${ }^{14}$ When $\omega \leqslant 1 \mathrm{MHz}$ (with all other parameters constant), the calculated IEDFs for all ions considered are virtually identical to each other. Thus, the in-trench charging potential distributions will also be identical and any differences in notch depth will be purely due to the additional bombardment of the sidewall by $\mathrm{He}^{+}$. 